\title{
Efficacy and safety of probiotics in the induction and maintenance of inflammatory bowel disease remission: a systematic review and meta-analysis
}

\author{
Mingshi Chen ${ }^{1}$, Yan Feng ${ }^{2}$, Wanli Liu ${ }^{1}$ \\ ${ }^{1}$ Department of Traditional Chinese Medicine, The First Hospital of China Medical University, Shenyang, China; ${ }^{2}$ Department of Internal Medicine, \\ North Hospital of Shenyang Fourth People's Hospital, Shenyang, China \\ Contributions: (I) Conception and design: M Chen; (II) Administrative support: Y Feng; (III) Provision of study materials or patients: W Liu; (IV) \\ Collection and assembly of data: All authors; (V) Data analysis and interpretation: All authors; (VI) Manuscript writing: All authors; (VII) Final \\ approval of manuscript: All authors. \\ Correspondence to: Wanli Liu. Department of Traditional Chinese Medicine, The First Hospital of China Medical University, Shenyang 110001, \\ China. Email: lwlsj2021@163.com.
}

Background Probiotics have been widely used in the treatment of inflammatory bowel disease (IBD) due to their special conditioning effects on the intestinal flora, but their efficacy in inducing and maintaining remission is still controversial. In the present study, we analyzed randomized controlled trials (RCTs) on the treatment of probiotics in IBD to systematically evaluate the efficacy of probiotics on the induction and maintenance of remission of IBD.

Methods: PubMed, Cochrane Central Register of Controlled Trials, Embase, Web of Science, Chinese Journal Full-text Database, Chinese Biomedical Literature Database, Wanfang, and Weipu databases were searched for RCTs on the treatment of probiotics in IBD induction and/or maintenance of remission. Two researchers independently extracted literature data and cross-checked the extracted data. The Cochrane bias risk assessment tool and the Jadad score were used to evaluate the quality of the literature. RevMan 5.20 software was used for the meta-analysis.

Results: A total of ten RCTs were included, which included 777 patients with ulcerative colitis (UC) and Crohn's disease (CD). Meta-analysis showed that there was no significant difference between the clinical recurrence rate of probiotics in the $\mathrm{CD}$ remission phase and the control group [relative risk (RR): 0.80, 95\% confidence interval (CI): 0.61-1.06, $\mathrm{P}=0.12]$. The clinical recurrence rate of $\mathrm{UC}$ in remission phase, the efficacy of probiotics compared with control group were not significantly different (RR: 1.07, 95\% CI: $0.80-1.42, \mathrm{P}=0.65)$. The induction remission rate of UC during the active phase, the efficacy of probiotics is better than that of the control group (RR: $1.47,95 \%$ CI: $1.09-1.98, \mathrm{P}=0.01$ ).

Discussion: In the present study, a systematic analysis of the efficacy of probiotics in CD and UC found that probiotics can induce remission during the active period of $\mathrm{UC}$, but have no obvious therapeutic advantage in maintaining CD and UC remission.

Keywords: Inflammatory bowel disease (IBD); Crohn's disease (CD); ulcerative colitis (UC); probiotics; metaanalysis

Submitted Sep 22, 2021. Accepted for publication Nov 12, 2021.

doi: 10.21037/apm-21-2996

View this article at: https://dx.doi.org/10.21037/apm-21-2996 


\section{Introduction}

Inflammatory bowel disease (IBD) is a chronic, non-specific intestinal inflammatory disease of unknown etiology, and includes ulcerative colitis (UC) and Crohn's disease (CD) (1). It is characterized by alternate symptom relief and recurrence. Its onset is believed to be mainly related to environmental factors, genetic susceptibility, intestinal flora disorders, and autoimmune dysfunction (2,3). Conventional treatment drugs for IBD include aminosalicylic acid preparations, hormones, immunosuppressants, and biological agents, such as tumor necrosis factor- $\alpha$ antibodies (4-6). Although the latter three types of drugs have definite curative effects, the adverse effects caused by long-term immunosuppression cannot be ignored (7).

Probiotics are a class of active microorganisms that are beneficial to the human body. They regulate and stabilize the intestinal environment, regulate the immune function of the intestinal mucosa and the permeability of the intestinal barrier, and improve the balance of intestinal flora and intestinal dysfunction. They are widely used in the treatment of IBD and can change the gut microbiome by enhancing the activity and/or size of the normal flora, thereby generating benefits $(8,9)$. For example, Lactobacillus is a more popular probiotic that is believed to secrete bacteriocins to prevent the adhesion of harmful bacteria translocation $(5,10)$. After entering the intestinal tract, probiotics will reoccupy or improve the environment around the intestinal tract, thereby stabilizing the $\mathrm{pH}$ value of the gastrointestinal tract, inhibiting the growth of harmful bacteria, and restoring the normal intestinal flora.

Although probiotics are living microorganisms, they can provide a variety of health benefits after consumption. An increasing number of clinical studies have explored the effect of probiotics on the induction and maintenance of IBD remission, but the results are controversial. In the present study, we conducted a meta-analysis to systematically evaluate this to provide a basis for the application of probiotics in the clinical treatment of IBD. We present the following article in accordance with the PRISMA reporting checklist (available at https://dx.doi. org/10.21037/apm-21-2996).

\section{Methods}

\section{Search strategy}

We searched the following international databasesPubMed, Cochrane Central Register of Controlled Trials,
Embase, and Web of Science-and Chinese databasesChinese Journal Full-text Database, Chinese Biomedical Literature Database, Wanfang, and Weipu database from June 2010 to June 2021 for relevant conference papers and materials. The following search terms were used: inflammatory bowel disease, IBD, ulcerative proctocolitis, ulcerative colitis, UC, Crohn's disease, CD, probiotics, Lactobacillus, Bifidobacterium, and Escherichia coli. Reference lists were analyzed for related literature.

\section{Inclusion criteria}

The inclusion criteria were as follows: (I) probiotics were used in the study of randomized controlled trials (RCTs) for IBD induction and/or the maintenance of remission; (II) the study participants were diagnosed with IBD by colonoscopy and biopsy pathological examination; (III) the trial included at least a probiotic group and a control group; (IV) measurable outcome indicators, such as induction of remission rate and clinical relapse rate. Duplicate studies were included the latest publication in this meta-analysis.

\section{Exclusion criteria}

The exclusion criteria were as follows: (I) review articles or case reports; (II) non-RCTs; (III) measurement methods and outcomes were not provided; (IV) study participants were pregnant or lactating; and (V) study participants had serious complications or other intestinal diseases with clear causes.

\section{Paper screening and data extraction}

We created a document extraction table, which included the first author, publication year, research objective, intervention measures, treatment course, outcome indicators, and adverse reactions. Two researchers independently screened the literature according to the inclusion and exclusion criteria, extracted the literature data, evaluated the quality of the literature, and cross-checked the extracted data. If consensus could not be reached between the two researchers, a third researcher was consulted. If the literature data in the studies were incomplete, the corresponding author of the article was contacted.

\section{Quality assessment}

We used the assessment risk of bias tool in the Cochrane 
Handbook for Systematic Reviews of Interventions to assess the quality of the literature. The items of bias tool included: whether the random allocation method was correct, whether the allocation concealment was implemented, the research objective, the implementer of the treatment plan, the result measurer, whether the statistician was blinded to the results, whether the research data results were complete, whether there was bias caused by selective reporting, and whether there were other biases. The Jadad score was used to evaluate the quality of the literature, and Jadad score $\geq 3$ points are considered that the quality of the literature is up to the standard, and the literature can be used for analysis.

\section{Statistical analysis}

RevMan 5.20 software provided by the Cochrane Collaboration was used for the meta-analysis, and the relative risk (RR) and $95 \%$ confidence interval (CI) of the count data were calculated. $\mathrm{P}<0.05$ indicated that the difference was statistically significant; $\mathrm{I}^{2}$ and $\chi^{2}$-test was used for the analysis of heterogeneity among the included studies, and a sensitivity analysis was carried out according to the Cochrane systematic review method. When $\mathrm{P}>0.10$ and $\mathrm{I}^{2}<50 \%$, there was no heterogeneity among the studies, and the fixed-effects model was used to merge the analysis result. If $\mathrm{P}<0.10$ and $\mathrm{I}^{2}>50 \%$, then the random-effects models was used to merge the analysis result. Subgroup analysis and sensitivity analysis were performed for studies with heterogeneity.

\section{Results}

\section{Search results and study characteristics}

A total of 1,427 articles were retrieved, including 896 in English and 529 in Chinese. Among them, 438 duplicate articles published use the same data or by different first authors were eliminated. After reading the title and abstract to exclude reviews, animal experiments, and non-RCTs, 174 articles were obtained. The full text was then read, and articles were screened according to the inclusion and exclusion criteria. Articles that were of low quality, had incomplete data, and non-RCTs were excluded. Ten RCTs were finally analyzed. The selection process is shown in Figure 1.

Among the ten articles available, there were $777 \mathrm{UC}$ and CD cases, of which 488 were UC and 289 are CD.
CD articles only involved reports related to recurrence rate, while $\mathrm{UC}$ has research on both recurrence rate and remission rate. All selected documents had a clear diagnosis and inclusion and exclusion criteria. The basic literature characteristics are shown in Table 1.

Eight papers used random number table method to generate random sequencing. There was no mention of allocation hiding in all articles. Seven papers used the double-blind method, seven described the risk of bias in blinded result assessment, all papers had complete data results and had no selective reporting bias, nine had lowrisk other biases, and one article had high-risk bias due to the small sample size. The detailed results are shown in Figure 2. The included study articles are scored by Jadad, five articles get 5 points, three articles get 4 points, and two articles get 3 points.

\section{Meta analysis results}

\section{CD recurrence rate}

Four articles studied the effect of probiotics on the recurrence rate of $\mathrm{CD}$. There were 145 cases with probiotics and 144 cases with other drugs. The analysis results were $\mathrm{P}=0.52$ and $\mathrm{I}^{2}=0 \%$, indicating homogeneity among the outcome indicators, so the fixed-effects model was used for combination analysis. The combined effect size RR: 0.80 and 95\% CI: 0.61-1.06, as shown in Figure 3. The result of the comprehensive effect size test was $Z=1.54$ and $\mathrm{P}=0.12$, so the meta-analysis results showed that the difference between probiotic intervention and other drug interventions was not statistically significant.

\section{$\mathrm{UC}$ recurrence rate}

Three articles were selected that studied the effects of probiotics on the recurrence rate of UC. A total of 140 cases treated with probiotics and 130 cases treated with other drugs were also included. The analysis results showed that $\mathrm{P}=0.19$ and $\mathrm{I}^{2}=40 \%$, indicating homogeneity between the outcomes, so the fixed-effects model was used for the combined analysis. The combined effect size was RR: 1.07 and 95\% CI: 0.80-1.42, as shown in Figure 4. The results of the comprehensive effect size test were $Z=0.45$ and $\mathrm{P}=0.65$. Therefore, the meta-analysis results showed that the effect of probiotics conditioning on the recurrence rate of UC did not change significantly compared with other drug treatments, and the difference was not statistically significant. 
Identification of studies via databases and registers

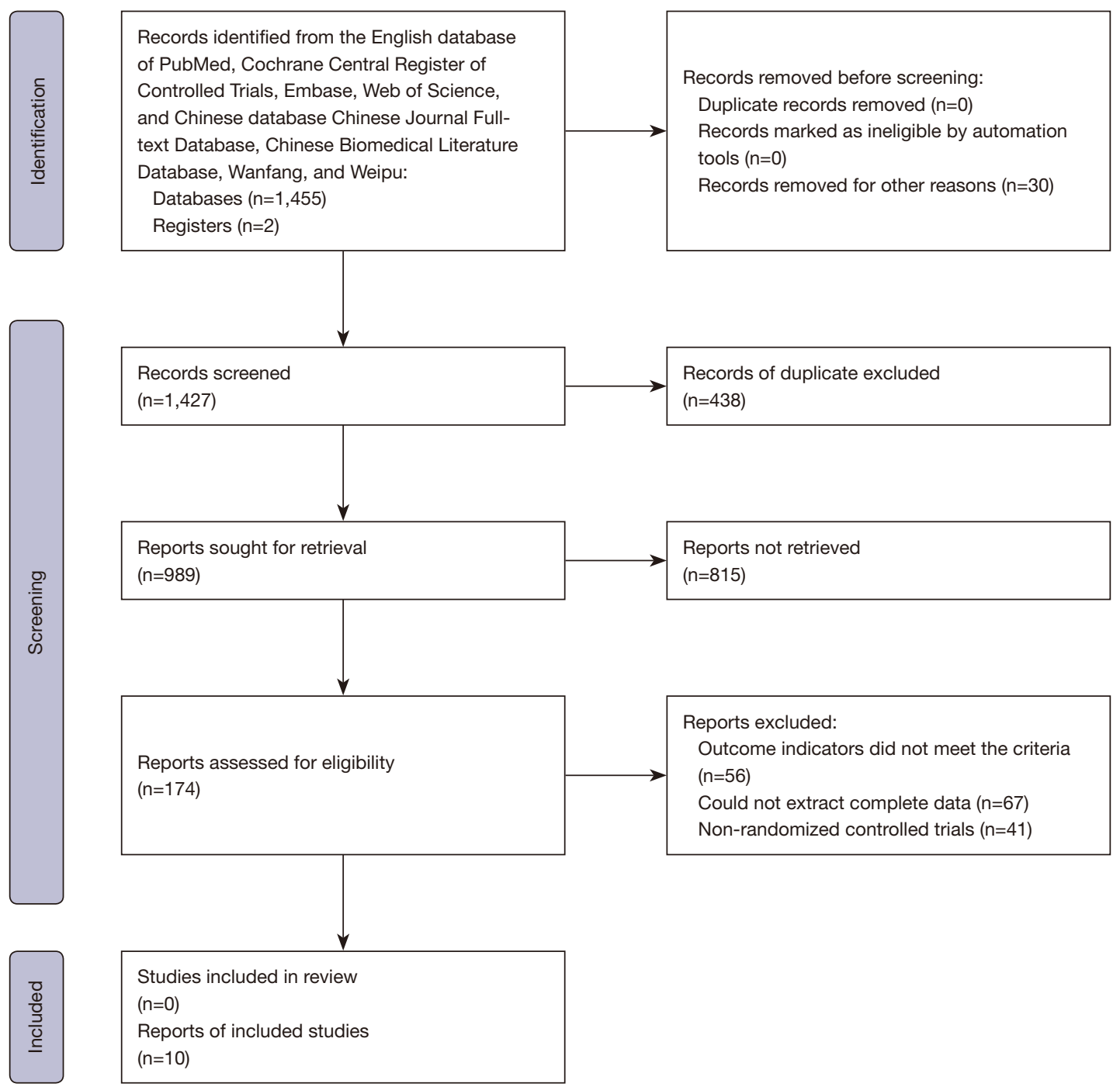

Figure 1 Research flowchart for the meta-analysis. CNKI, Chinese Journal Full-text Database.

\section{UC-induced remission rate}

Three articles were selected to study the use of probiotics to induce UC remission. A total of 109 cases induced by probiotics and 109 cases induced by other drugs were included. The analysis results showed that $\mathrm{P}=0.29$ and $\mathrm{I}^{2}=18 \%$, indicating homogeneity between the results, and the fixed-effects model was used for the analysis. After the analysis, the effect size was RR: 1.47 and $95 \%$ CI: 1.09-1.98, as shown in Figure 5. The result of the effect size test was $\mathrm{Z}=2.53$ and $\mathrm{P}=0.01$. Therefore, as seen in the results of the meta-analysis, the remission effect induced by probiotics on
UC was higher than that induced by other drugs, and the difference was statistically significant.

\section{Publication bias}

For the analysis of the efficacy of probiotics on maintaining $\mathrm{CD}$ and $\mathrm{UC}$ remission, and inducing remission during UC active period, the number of articles included in the above three indicators analyzed in this study was less than the publication bias requirement $(<5)$, so publication bias analysis was not performed. 
Table 1 Basic characteristics of the included articles

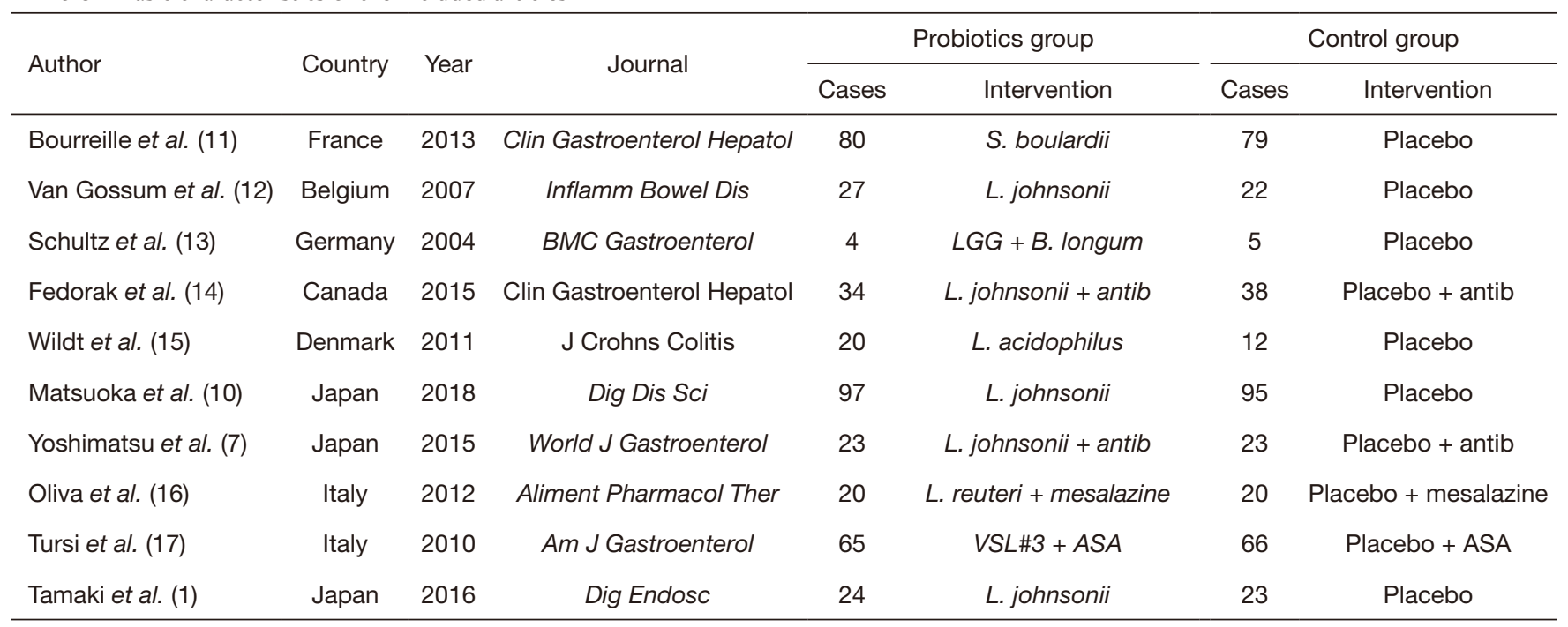

S. boulardii, Salmonella. Boulardii; L. johnsonii, Lactobacillus johnsonii; LGG, Lactobacillus. rhamnosus GG; B. longum, Bifidobacterium longum; Antib, antibacterials; L. acidophilus, Lactobacillus. acidophilus; L. reuteri, Lactobacillus. reuteri; VSL\#3, probiotics mixture; ASA, aminosalicylic acid.

\section{Risk of bias}

Among the eligible studies, eight found that random sequencing generation was low risk $(1,7,11-14,16,17)$ and 2 were unclear $(10,15)$. All studies on the allocation of hidden risk of bias were unclear. Seven studies had a low risk of bias for blinding participants and researchers $(1,7,11-14,17)$ and three studies had a unclear risk of bias for blinding participants and researchers $(10,15,16)$. Seven studies described the low risk of bias in blinded results assessment $(1,7,11-14,17)$ and three trials were unknown $(10,15,16)$. All studies had a low risk of bias for incomplete outcome data. For the selective reporting domains, all studies were judged to have a low risk of bias and one study had a high risk of other bias due to a small sample size (13), as shown in Figure 6.

\section{Discussion}

As a chronic relapsing disease, the clinical course of IBD is often characterized by alternating episodes and remissions. Therefore, inducing remission in the active period and avoiding recurrence in the remission period are key for the treatment of IBD. In the present study, we systematically evaluated the efficacy of probiotics on IBD. A meta-analysis of ten RCTs related to the induction and maintenance of IBD remission by probiotics showed that, although probiotics have no significant effect on reducing the recurrence rate of $\mathrm{CD}$ and $\mathrm{UC}$, their effects are similar to mesalazine, but probiotics have a significant effect on alleviating the clinical symptoms of $\mathrm{UC}$ and improving the remission of UC (10).

However, a recent double-blind RCT found that probiotics did not show superiority compared with placebo in the induction of remission during the active phase of $\mathrm{UC}$, and the induced remission rate was even lower than that of placebo (54\% vs. 89\%) (18). This negative effect of probiotics was due to the probiotic preparation of Escherichia coli Nissle 1917 used in the study, which could promote the growth of pathogenic Escherichia coli, which may be associated with IBD in a symbiotic manner, and these patients who had negative effects of probiotic treatment started treatment without using antibacterial drugs to kill Escherichia coli $(18,19)$. In addition, a subgroup analysis found that probiotics are as effective as mesalazine in maintaining $\mathrm{UC}$ remission. In recent years, there have been many studies on the efficacy of probiotic preparations for UC $(1,5,6,9,10,14,20)$. In the present study, we screened ten high-quality RCTs and analyzed the induction of remission during the active phase and maintenance of remission during the remission phase. With increasing research on the pathophysiology of IBD, more and more evidence indicates that the imbalance of intestinal flora and invasive and protective strains may be stimulated 
by antigens, abnormal intestinal mucosal immune function, intestinal epithelial cell metabolism, and changes in intestinal wall permeability, which play an important role in the occurrence and development of IBD. The imbalance of

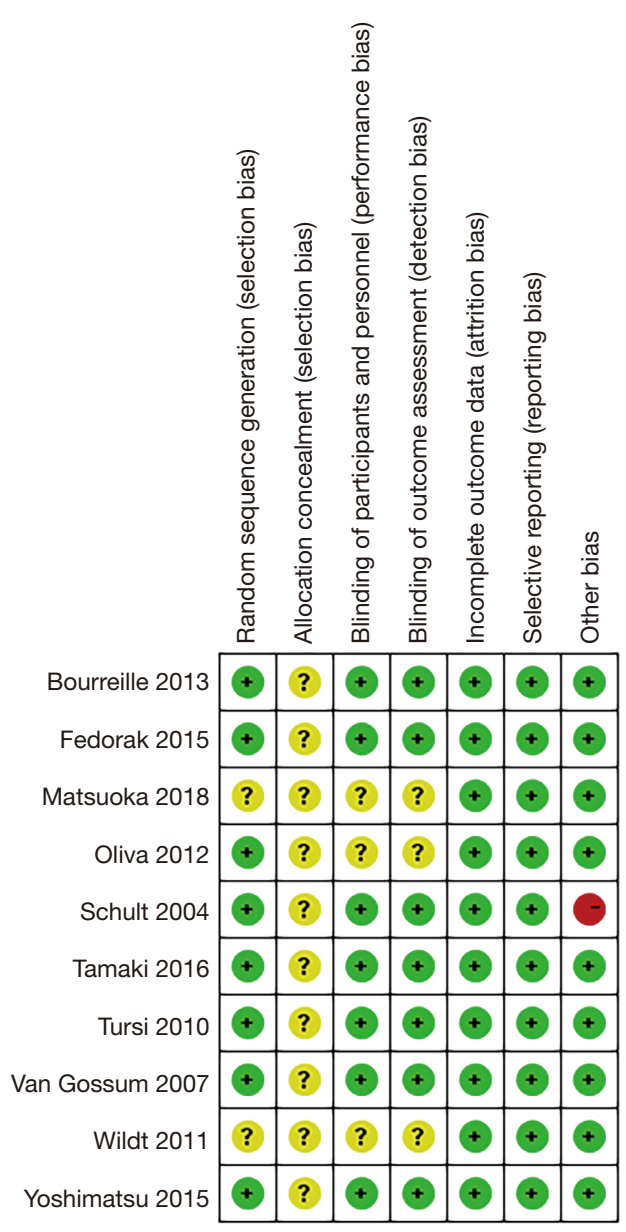

Figure 2 Literature quality evaluation. intestinal flora causes the decline of the intestinal epithelial barrier function, the overexpression of pro-inflammatory mediators, and the lack of protective signals. A series of excessive immune responses eventually lead to the occurrence of IBD $(11,18,21)$.

Therefore, the application of probiotics to regulate or restore the balance of intestinal flora is a new treatment for IBD (1). Its mechanism of action may include regulating the intestinal immune response and the composition and activity of the intestinal flora, and improving the intestinal barrier function $(19,22)$. Inhibiting the adhesion of pathogenic bacteria in the intestine to intestinal epithelial cells reduces intestinal epithelial cell damage, prevents bacterial translocation, and avoids the activation of intestinal immune cells. It also improves the physical barrier function of the intestinal epithelium by regulating the connections between intestinal epithelial cells. However, our research results show that probiotics induce remission during the active phase of UC and have a good effect on CD, but we have not found a clear advantage in maintaining $\mathrm{UC}$ remission. Because the regulation mechanism and ability of each probiotic is different, and the subgroups analyzed are different, the results of this study are different from the conclusions of similar studies (23). So we recommend that patients take probiotics as an adjunct to the treatment of IBD, and develop a personalized treatment plan based on the specific situation of the patient, rather than a standard for all patients.

The limitations of this meta-analysis are mainly that the types, dosages, usage, and duration of probiotic preparations were different among the studies, and the efficacy evaluation indicators were not consistent. Differences in the above treatment plans and evaluation indicators may affect the final evaluation results. In the future, RCTs with large sample sizes, high quality, and consistent evaluation standards need

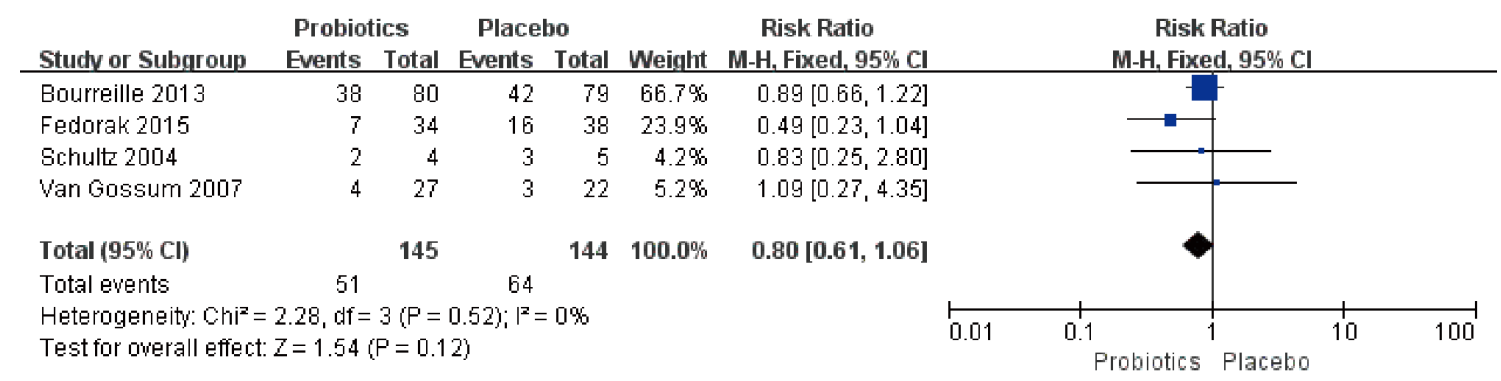

Figure 3 Forest plot of Crohn's disease recurrence rate using a fixed-effects model. Comparison of probiotics application and other drug applications. Statistical method: Mantel-Haenszel of fixed-effects model [relative risk and 95\% confidence interval (CI)]. 


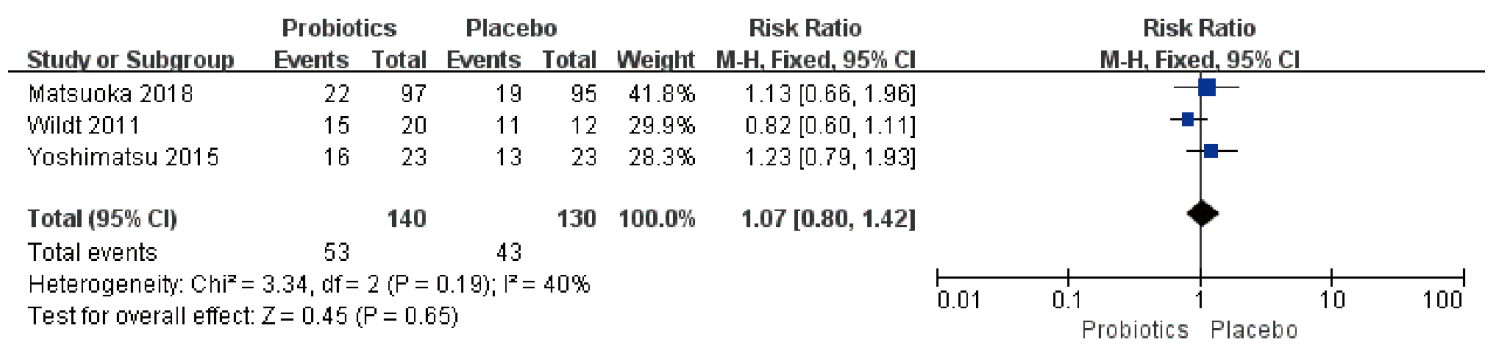

Figure 4 Forest plot of recurrence rate of ulcerative colitis (UC). Comparison of the recurrence rate of UC after conditioning with probiotics and the recurrence rate after treatment with other drugs. Statistical method: Mantel-Haenszel of the fixed-effects model [relative risk and $95 \%$ confidence interval $(\mathrm{CI})]$.

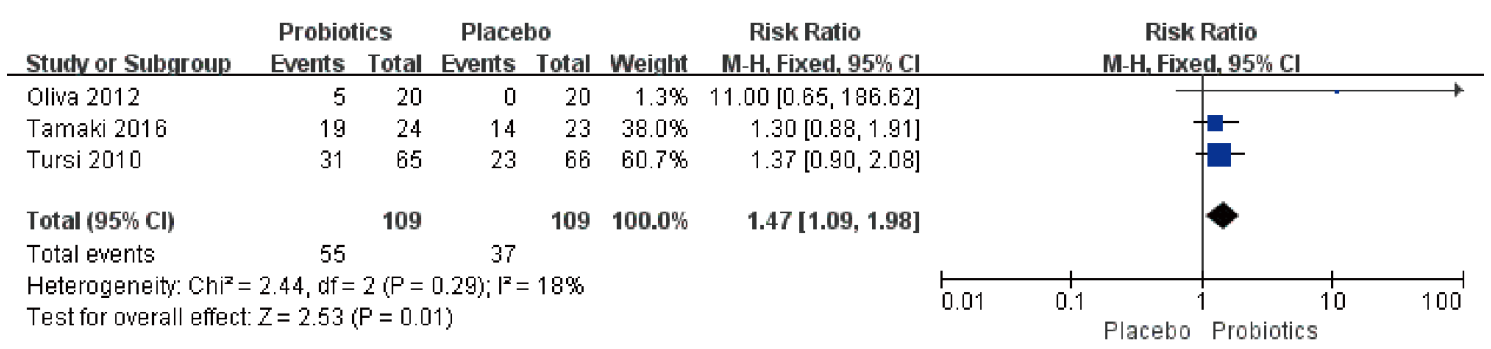

Figure 5 Forest plot of ulcerative colitis-induced remission rate. Comparison of probiotics and other drugs to induce remission rates. Statistical method: Mantel-Haenszel of the fixed effects model [relative risk and $95 \%$ confidence interval (CI)].

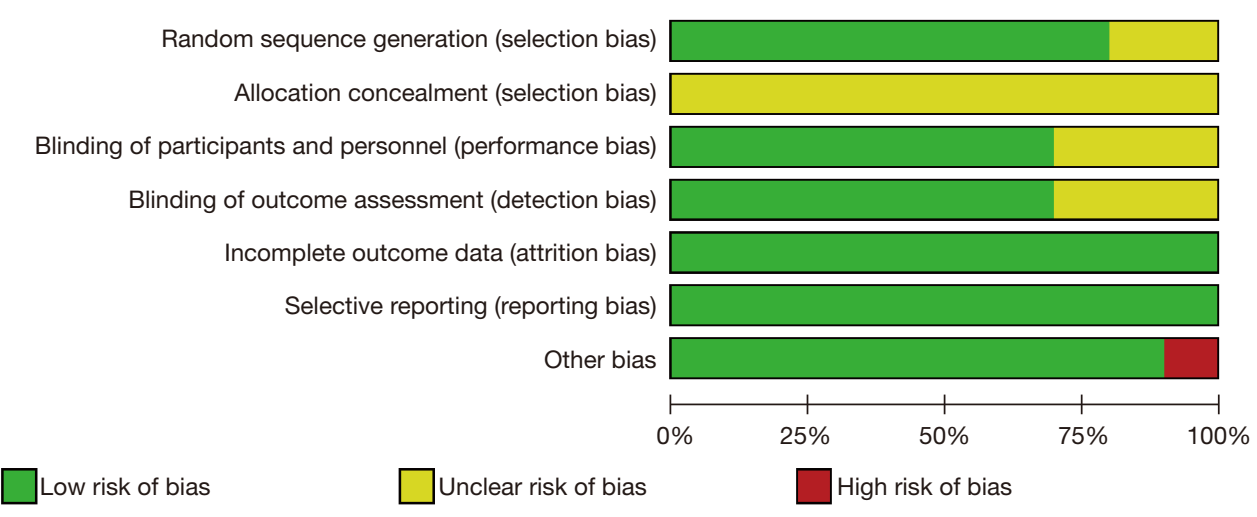

Figure 6 Intensity and distribution of the quality risk of the articles included in the study.

to be carried out to clarify the efficacy of probiotics in the induction and maintenance of IBD remission.

\section{Conclusions}

In the present study, we conducted a systematic analysis of probiotics in the active phase of CD and UC to induce remission and maintain the remission period to reduce the recurrence rate. We found that probiotics induce remission during the active phase of UC and have a good effect on CD. The maintenance of UC remission was not found to have a therapeutic advantage.

\section{Acknowledgments}

Funding: None. 


\section{Footnote}

Reporting Checklist: The authors have completed the PRISMA reporting checklist. Available at https://dx.doi. org/10.21037/apm-21-2996

Conflicts of Interest: All authors have completed the ICMJE uniform disclosure form (available at https://dx.doi. org/10.21037/apm-21-2996). The authors have no conflicts of interest to declare.

Ethical Statement: The authors are accountable for all aspects of the work in ensuring that questions related to the accuracy or integrity of any part of the work are appropriately investigated and resolved.

Open Access Statement: This is an Open Access article distributed in accordance with the Creative Commons Attribution-NonCommercial-NoDerivs 4.0 International License (CC BY-NC-ND 4.0), which permits the noncommercial replication and distribution of the article with the strict proviso that no changes or edits are made and the original work is properly cited (including links to both the formal publication through the relevant DOI and the license). See: https://creativecommons.org/licenses/by-nc-nd/4.0/.

\section{References}

1. Tamaki H, Nakase H, Inoue S, et al. Efficacy of probiotic treatment with Bifidobacterium longum 536 for induction of remission in active ulcerative colitis: A randomized, double-blinded, placebo-controlled multicenter trial. Dig Endosc 2016;28:67-74.

2. Devkota S, Wang Y, Musch MW, et al. Dietary-fatinduced taurocholic acid promotes pathobiont expansion and colitis in Il10-/- mice. Nature 2012;487:104-8.

3. Frank DN, Robertson CE, Hamm CM, et al. Disease phenotype and genotype are associated with shifts in intestinal-associated microbiota in inflammatory bowel diseases. Inflamm Bowel Dis 2011;17:179-84.

4. Iheozor-Ejiofor Z, Kaur L, Gordon M, et al. Probiotics for maintenance of remission in ulcerative colitis. Cochrane Database Syst Rev 2020;3:CD007443.

5. Limketkai BN, Iheozor-Ejiofor Z, Gjuladin-Hellon T, et al. Dietary interventions for induction and maintenance of remission in inflammatory bowel disease. Cochrane Database Syst Rev 2019;2:CD012839.

6. Saez-Lara MJ, Gomez-Llorente C, Plaza-Diaz J, et al.
The role of probiotic lactic acid bacteria and bifidobacteria in the prevention and treatment of inflammatory bowel disease and other related diseases: a systematic review of randomized human clinical trials. Biomed Res Int 2015;2015:505878.

7. Yoshimatsu Y, Yamada A, Furukawa R, et al. Effectiveness of probiotic therapy for the prevention of relapse in patients with inactive ulcerative colitis. World J Gastroenterol 2015;21:5985-94.

8. Bjarnason I, Sission G, Hayee B. A randomised, doubleblind, placebo-controlled trial of a multi-strain probiotic in patients with asymptomatic ulcerative colitis and Crohn's disease. Inflammopharmacology 2019;27:465-73.

9. Jakubczyk D, Leszczyńska K, Górska S. The Effectiveness of Probiotics in the Treatment of Inflammatory Bowel Disease (IBD)-A Critical Review. Nutrients 2020;12:1973.

10. Matsuoka K, Uemura Y, Kanai T, et al. Efficacy of Bifidobacterium breve Fermented Milk in Maintaining Remission of Ulcerative Colitis. Dig Dis Sci 2018;63:1910-9.

11. Bourreille A, Cadiot G, Le Dreau G, et al. Saccharomyces boulardii does not prevent relapse of Crohn's disease. Clin Gastroenterol Hepatol 2013;11:982-7.

12. Van Gossum A, Dewit O, Louis E, et al. Multicenter randomized-controlled clinical trial of probiotics (Lactobacillus johnsonii, LA1) on early endoscopic recurrence of Crohn's disease after lleo-caecal resection. Inflamm Bowel Dis 2007;13:135-42.

13. Schultz M, Timmer A, Herfarth HH, et al. Lactobacillus GG in inducing and maintaining remission of Crohn's disease. BMC Gastroenterol 2004;4:5.

14. Fedorak RN, Feagan BG, Hotte N, et al. The probiotic VSL\#3 has anti-inflammatory effects and could reduce endoscopic recurrence after surgery for Crohn's disease. Clin Gastroenterol Hepatol 2015;13:928-35.e2.

15. Wildt S, Nordgaard I, Hansen U, et al. A randomised double-blind placebo-controlled trial with Lactobacillus acidophilus La-5 and Bifidobacterium animalis subsp. lactis BB-12 for maintenance of remission in ulcerative colitis. J Crohns Colitis 2011;5:115-21.

16. Oliva S, Di Nardo G, Ferrari F, et al. Randomised clinical trial: the effectiveness of Lactobacillus reuteri ATCC 55730 rectal enema in children with active distal ulcerative colitis. Aliment Pharmacol Ther 2012;35:327-34.

17. Tursi A, Brandimarte G, Papa A, et al. Treatment of relapsing mild-to-moderate ulcerative colitis with the probiotic VSL\#3 as adjunctive to a standard pharmaceutical treatment: a double-blind, randomized, placebo-controlled 
study. Am J Gastroenterol 2010;105:2218-27.

18. Petersen AM, Mirsepasi H, Halkjær SI, et al. Ciprofloxacin and probiotic Escherichia coli Nissle add-on treatment in active ulcerative colitis: a double-blind randomized placebo controlled clinical trial. J Crohns Colitis 2014;8:1498-505.

19. Leccese G, Bibi A, Mazza S, et al. Probiotic Lactobacillus and Bifidobacterium Strains Counteract Adherent-Invasive Escherichia coli (AIEC) Virulence and Hamper IL-23/ Th17 Axis in Ulcerative Colitis, but Not in Crohn's Disease. Cells 2020;9:1824.

20. White R, Atherly T, Guard B, et al. Randomized, controlled trial evaluating the effect of multi-strain probiotic on the mucosal microbiota in canine idiopathic inflammatory bowel disease. Gut Microbes 2017;8:451-66.
21. Rossi G, Pengo G, Caldin M, et al. Comparison of microbiological, histological, and immunomodulatory parameters in response to treatment with either combination therapy with prednisone and metronidazole or probiotic VSL\#3 strains in dogs with idiopathic inflammatory bowel disease. PLoS One 2014;9:e94699.

22. Dong LN, Wang M, Guo J, et al. Role of intestinal microbiota and metabolites in inflammatory bowel disease. Chin Med J (Engl) 2019;132:1610-4.

23. Jia K, Tong X, Wang R, et al. The clinical effects of probiotics for inflammatory bowel disease: A metaanalysis. Medicine (Baltimore) 2018;97:e13792.

(English Language Editor: R. Scott)
Cite this article as: Chen M, Feng Y, Liu W. Efficacy and safety of probiotics in the induction and maintenance of inflammatory bowel disease remission: a systematic review and meta-analysis. Ann Palliat Med 2021;10(11):11821-11829. doi: 10.21037/apm-21-2996 